\title{
Risco de Recorrência do Carcinoma Hepatocelular após o Uso de Antivirais de Ação Direta no Tratamento de Hepatite C: Revisão Sistemática e Metanálise
}

\author{
doi: https://doi.org/10.32635/2176-9745.RBC.2021v67n2.1220 \\ Risk of Hepatocellular Carcinoma Recurrence After the Use of Direct-Acting Antivirals in the Treatment of Hepatitis C:Systematic \\ Review and Metanalysis \\ Riesgo de Recurrencia del Carcinoma Hepatocelular tras el Uso de Antivirales de Acción Directa en el Tratamiento de la \\ Hepatitis C: Revisión Sistemática y Metanálisis
}

Jefferson Wrublack Cuba'; Amanda da Silva Anjos ${ }^{2}$; Edimar Leandro Toderke ${ }^{3}$; Filipe Vieira Kwiatkowski ${ }^{4}$

RESUMO

Introduçáo: A hepatite $\mathrm{C}$ está associada ao desenvolvimento do carcinoma hepatocelular ( $\mathrm{CHC}$ ). $\mathrm{O}$ regime terapêutico baseado em interferon vem sendo substituído pelos antivirais de ação direta (AAD) para tratamento da infecção pelo vírus da hepatite $\mathrm{C}$ (HCV). Contudo, estudos recentes evidenciaram um aumento inesperado da recorrência do $\mathrm{CHC}$ em pacientes tratados com $\mathrm{AAD}$ para resolução da hepatite C. Objetivo: Avaliar o risco de recorrência de hepatocarcinoma após uso de AAD em pacientes com infecção por HCV. Método: Realizou-se um levantamento nas bases de dados PubMed, MEDLINE e LILACS de acordo com os descritores DeCS/MeSH ((hepatocellular carcinoma) AND recurrence) AND Direct-acting antiviral. A revisão obedeceu ao protocolo PRISMA e está cadastrada na plataforma PROSPERO. A análise estatística dos dados foi realizada no software RStudio. Resultados: Sete artigos foram selecionados resultando em 847 pacientes. Entre os tratados com AAD, a taxa de recorrência variou entre 11,1\% e 42,1\% e, no grupo controle, ocorreu em 5\% a 65,6\% dos pacientes. O risco relativo (RR) de recorrência do CHC no grupo de pacientes que recebeu AAD foi menor do que o risco evidenciado no grupo controle, apesar de náo haver significância estatística (RR 0,71 95\% IC [0,55;0,93] I²=38\%, p=0,14). O tempo até o diagnóstico da recorrência teve uma média de 9,35 meses no grupo exposto à terapia e 13,42 meses no grupo controle. Conclusáo: Sugere-se que a terapia com $\mathrm{AAD}$ não aumenta o risco de recorrência do $\mathrm{CHC}$ em comparação com grupos controle. Nos pacientes que desenvolveram recorrência, ocorreu com maior frequência dentro do primeiro ano após introdução dos AAD.

Palavras-chave: Carcinoma Hepatocelular/etiologia; Recidiva; Antivirais; Hepatite C/complicaçóes.

\section{ABSTRACT}

Introduction: Hepatitis $C$ is associated with the development of hepatocellular carcinoma (HCC). The interferon-based therapeutic regimen has been replaced by direct-acting antivirals (AAD) to treat HCV virus infection. However, recent studies have shown an unexpected increase in HCC recurrence in patients treated with AAD to resolve hepatitis C. Objective: To assess the risk of hepatocarcinoma recurrence after using $\mathrm{AAD}$ in patients with $\mathrm{HCV}$ infection. Method: A survey was carried out in PubMed, MEDLINE, and LILACS databases according to the descriptors $\mathrm{DeCS} / \mathrm{MeSH}$ ((hepatocellular carcinoma) AND recurrence) AND Directacting antiviral. The review followed the PRISMA protocol and is registered on the PROSPERO platform. The data statistical analysis was performed through RStudio software. Results: Seven articles were selected resulting in 847 patients. Among those treated with AAD, the recurrence rate varied between $11.1 \%$ to $42.1 \%$ and, in the control group, it occurred in $5 \%$ to $65.6 \%$ of the patients. The relative risk (RR) of recurrence of HCC in the group of patients who received AAD was less than the risk evidenced in the control group, although there is no statistical significance (RR 0.71 $95 \%$ CI $\left.[0.55 ; 0.93] \mathrm{I}^{2}=38 \%, \mathrm{p}=0.14\right)$. The mean time until the diagnosis of recurrence was 9.35 months in the group exposed to therapy and 13.42 months in the control group. Conclusion: It is suggested that therapy with AAD does not increase the risk of HCC recurrence compared to control groups. In patients who developed recurrence, it occurred more frequently within the first year after the introduction of AAD.

Key words: Carcinoma, Hepatocellular/etiology; Recurrence; Antiviral Agents; Hepatitis C/complications.

\section{RESUMEN}

Introducción: La hepatitis $\mathrm{C}$ está asociada con el desarrollo de carcinoma hepatocelular $(\mathrm{CHC})$. El régimen terapéutico basado en interferón ha sido reemplazado por antivirales de acción directa $(\mathrm{AAD})$ para tratar la infección por VHC. Sin embargo, estudios recientes han mostrado un incremento inesperado en la recurrencia del $\mathrm{CHC}$ en pacientes tratados con $\mathrm{AAD}$ para resolución de la hepatitis C. Objetivo: Evaluar el riesgo de recurrencia del hepatocarcinoma después de usar AAD en pacientes con infección por VHC. Método: Se realizó una pesquisa en las bases de datos PubMed, MEDLINE y LILACS según los descriptores DeCS/MeSH ((carcinoma hepatocelular) AND recurrencia) AND antiviral de acción directa. La revisión siguió el protocolo PRISMA y está registrada en la plataforma PROSPERO. El análisis estadístico de los datos se realizó mediante el software RStudio. Resultados: Fueron seleccionados 7 artículos resultando en 847 pacientes. Entre los tratados con AAD, la tasa de recurrencia varió entre el 11,1\% y el $42,1 \%$ y, en el grupo de control, ocurrió entre el 5\% y el 65,6\% de los pacientes. El riesgo relativo (RR) de recurrencia del $\mathrm{CHC}$ en el grupo de pacientes que recibieron $\mathrm{AAD}$ fue inferior que el riesgo evidenciado en el grupo control, aunque no hay significación estadística (RR 0,71; IC del 95\% $\left.[0,55 ; 0,93] \mathrm{I}^{2}=38 \%, \mathrm{p}=0,14\right)$. El tiempo hasta el diagnóstico de recidiva fue de 9,35 meses en el grupo expuesto a terapia y de 13,42 meses en el grupo control. Conclusión: Se sugiere que la terapia con $\mathrm{AAD}$ no aumenta el riesgo de recurrencia del CHC en comparación con los grupos control. En los pacientes que desarrollaron recurrencia, esta ocurrió con mayor frecuencia durante el primer año después de la introducción de los AAD. Palabras clave: Carcinoma Hepatocelular/etiología; Recurrencia; Antivirales; Hepatitis C/complicaciones.

1,2Faculdades Pequeno Príncipe. Curitiba (PR), Brasil. E-mails: jef_cub@hotmail.com; anjosacademico@outlook.com. Orcid iD: https://orcid.org/0000-0002-0425-4564; Orcid iD: https://orcid.org/0000-0002-8064-5161

${ }^{3}$ Hospital e Maternidade Municipal de São José dos Pinhais. São José dos Pinhais (PR), Brasil. E-mail: edimar.toderke@fpp.edu.br. Orcid iD: https://orcid. org/0000-0002-2790-2173

${ }^{4}$ Hospital Santa Casa de Misericórdia de Pelotas. Pelotas (RS), Brasil. E-mail: filipevk79@yahoo.com.br. Orcid iD: https://orcid.org/0000-0002-9965-7381

Endereço para correspondência: Jefferson Wrublack Cuba. Rua Rui Barbosa, 1410 - Centro. Campo Largo (PR), Brasil. CEP 83601-140. E-mail: jef_cub@hotmail.com 


\section{INTRODUÇÃO}

A infecção pelo vírus da hepatite C (HCV) é a segunda hepatite viral mais comum no Brasil. No período de 1999 a 2018, foram notificados 228.695 casos de hepatite C no país, correspondendo a $36,1 \%$ do total de casos de hepatites virais ${ }^{1}$. É uma importante causa de morbidade e mortalidade, sendo a principal causa de doença hepática crônica e associada a diversas complicaçóes ${ }^{2,3}$. No que diz respeito à mortalidade, está relacionada a $76 \%$ dos casos de morte por hepatite viral no Brasil ${ }^{1}$.

A hepatite C está fortemente associada ao desenvolvimento do carcinoma hepatocelular $(\mathrm{CHC})$, aumentando o risco de desenvolvimento dessa neoplasia em torno de 15 a 20 vezes $^{3,4}$. Quando o processo de cirrose está estabelecido, a taxa de desenvolvimento de $\mathrm{CHC}$ gira em torno de $1 \%$ a $4 \%$ anualmente ${ }^{3}$. Em 2020, o número estimado de novos casos de neoplasias hepáticas no Brasil foi de $12.674^{5}$, sendo a sexta causa de mortalidade por câncer em homens e a oitava causa em mulheres no país ${ }^{6}$. Além do $\mathrm{CHC}$, a hepatite $\mathrm{C}$ está relacionada à insuficiência hepática e à descompensação de cirrose, além de diversas alteraçôes extra-hepáticas, sendo uma significativa demanda para o transplante hepático².

$\mathrm{O}$ tratamento da infecçáo pelo HCV tem como objetivo eliminar a infecção, diminuir as taxas de transmissibilidade e reduzir o risco de desenvolvimento do hepatocarcinoma ${ }^{3}$. Até recentemente, a terapia anti-HCV era baseada em regimes contendo interferon (IFN), que garantiam a cura ou a resposta virológica sustentada (RVS)/HCV-RNA indetectável a partir de 12 semanas após o término do tratamento) ${ }^{7}$ em aproximadamente $50 \%$ dos pacientes. Observou-se que, nos pacientes que alcançavam a RVS, havia redução da incidência do $\mathrm{CHC}^{4}$, bem como redução da recorrência do $\mathrm{CHC}$ após terapia curativa. $\mathrm{O}$ mecanismo desse fenômeno, contudo, é incerto, podendo ser associado à RVS ou ao efeito antitumoral imunomediado do IFN ${ }^{8,9}$.

O IFN- $\alpha$ é uma citocina que possui atividade antiviral, imunomoduladora e antiproliferativa, por meio da inibição da transcrição, tradução, processamento pós-tradução, maturação e liberação viral ${ }^{8}$. Apresenta melhores respostas quando combinado com ribavirina (análogo da guanosina) e com um processo de peguilação (adição de uma molécula de polietilenoglicol à molécula de IFN, visando a estender sua meia-vida, permitindo assim a administração do fármaco semanalmente) $)^{8,10}$.

Apesar disso, os esquemas com IFN apresentam alta toxicidade, principalmente hematológica, autoimune, neurológica e psiquiátrica, além de sintomas como febre, calafrios, cefaleia, mialgia, artralgia, náuseas, vômitos e diarreia, que ocorrem após a administração intravenosa do IFN. Ademais, a duração prolongada do tratamento, a administraçáo intravenosa, o alto custo e a consequente menor adesão levaram à descontinuaçáo desse tratamento $^{8,11}$. Por conta disso, passou-se a buscar novas terapias que pudessem substituir os esquemas baseados em IFN ${ }^{4}$.

Entre as inovações conquistadas na área, uma delas foi a descoberta dos antivirais de açáo direta (AAD), que melhoraram o perfil de tolerabilidade dos pacientes e alcançaram taxas de cura do HCV em mais de $90 \%$ dos pacientes ${ }^{4}$. Os $\mathrm{AAD}$ são fármacos que atuam em alvos moleculares definidos. Existem três classes principais de AAD: inibidores de protease NS3 (boceprevir, telaprevir, simepravir, asunaprevir, paritaprevir e grazoprevir); inibidores de NS5A (daclatasvir, ledispasvir, ombitasvir, elbasvir e velpatasvir); e inibidores de polimerase NS5B (sofosbuvir e dasabuvir). Esses medicamentos atuam impedindo o processamento, a replicação e a montagem viral $^{10,12}$.

Entretanto, por ser uma terapia recente, são pouco conhecidos seus efeitos a médio e longo prazo. Alguns estudos sugerem que os $\mathrm{AAD}$ possuem benefícios no tratamento do $\mathrm{HCV}$, como a capacidade de reduzir o gradiente de pressão venosa hepática em pacientes cirróticos com hipertensão portal, por causa de modificaçóes na consistência hepática ${ }^{4}$, bem como melhoria na função hepática e diminuição do risco de incidência de CHC. Outros benefícios incluem a dispensação da coadministração de ribavirina na maioria dos casos (exceto em pacientes com cirrose descompensada) e duração do tratamento diminuída para oito a 12 semanas apenas, muito inferior quando comparada com a duração da terapia com IFN ${ }^{9,13-15}$.

Ademais, os AAD são administrados via oral e apresentam menor quantidade de efeitos adversos significativos, sendo bem tolerados pela maior parte dos pacientes ${ }^{14}$. Os mais comuns correspondem à: cefaleia, fadiga, náusea e diarreia, e ocorrem em $\geq 1 / 10$ pacientes; deve-se monitorar também os níveis da enzima transaminase glutâmico-pirúvica (TGP) e bilirrubinas, que podem se elevar durante tratamento com $\mathrm{AAD}^{16}$.

No entanto, outros estudos trouxeram à evidência um possível favorecimento da recorrência do $\mathrm{CHC}$ nos pacientes previamente curados e tratados com AAD. Essa hipótese, todavia, não é consenso dentro do meio científico, já que alguns estudos não demonstram tal relaçáo ${ }^{4}$.

Dessa forma, o presente estudo visa a avaliar o risco de recorrência de hepatocarcinoma após uso de AAD em pacientes com infecçáo por HCV. Elaborou-se a seguinte pergunta norteadora: $\mathrm{O}$ risco de recorrência de hepatocarcinoma aumenta após uso de $\mathrm{AAD}$ em pacientes com infecção por HCV? 


\section{MÉTODO}

O presente estudo caracteriza-se por ser uma revisão do tipo sistemática, com metanálise, tendo sido norteado por uma pergunta de pesquisa formulada por meio dos critérios PICO (P: população/pacientes; I: intervenção; C: comparação/controle; O: desfecho/outcome). Ademais, houve adoçâo do checklist Preferred Reporting Items for Systematic Review and Meta-analysis Statement (PRISMA) para construção metodológica ${ }^{17}$. Por fim, a atual revisão sistemática consta registrada no sistema International Prospective Register of Systematic Reviews (PROSPERO) com objetivo de náo duplicaçóes de artigos científicos (ID: CRD42020182702).

Os critérios de elegibilidade adotados foram publicações dos últimos cinco anos (janeiro de 2015 a abril de 2020), ensaios clínicos randomizados controlados, estudos caso-controle, estudos de coorte prospectiva (ou concorrente), ensaios que envolvessem pacientes já acometidos por $\mathrm{CHC}$ em remissão, pacientes com infecção por $\mathrm{HCV}$ e pacientes que foram submetidos à terapia $\mathrm{AAD}$ por no mínimo três meses em qualquer dose ou esquema. Para exclusão, os critérios foram artigos que correlacionavam o papel dos $\mathrm{AAD}$ apenas com o primeiro evento neoplásico e estudos com participantes de idade inferior a 18 anos.

A identificação dos estudos foi realizada nas bases de dados MEDLINE, LILACS e PubMed com o uso dos descritores DeCS/MeSH: ((hepatocellular carcinoma) AND recurrence) AND Direct-acting antiviral. A seleção dos artigos foi realizada do seguinte modo: busca em bases de dados, exclusão de estudos duplicados, revisão de título e resumo e adequaçáo aos critérios de elegibilidade.

Após a seleçáo dos estudos, estes foram tabelados conforme autoria, título, ano de publicação, local de publicação e periódico publicado. Posteriormente, foram analisados e tabelados de acordo com objetivo de pesquisa, métodos e resultados obtidos.

Para a avaliação de riscos de viés, realizada pelos autores que analisaram independentemente os ensaios selecionados, utilizou-se a ferramenta Risk of Bias in Non-randomised Studies of Interventions (ROBINS-I), desenvolvida pela Cochrane. A ferramenta ROBINS-I avalia o risco de viés geral dos estudos de intervenção não randomizados por meio de alguns domínios: 2 pré- -intervenção (viés de domínios de confusão e de seleção), 1 durante intervenção (viés de classificação de intervençôes) e 4 pós-intervenção (viés de desvios de intervençôes, dados ausentes, desfecho e seleção de resultados relatados). Esses domínios são avaliados por intermédio de questionamentos presentes na ferramenta, que auxiliam no julgamento de viés. As respostas a esses questionamentos (sim/provavelmente sim/ provavelmente não/não/sem informação) são responsáveis por prover à base para o julgamento do nível de viés de cada domínio, que pode ser baixo, moderado, severo, crítico ou sem informaçấo. O risco de viés geral se dá pela avaliação combinada dos riscos de viés dos domínios. Um baixo risco de viés geral ocorre quando todos os domínios apresentam baixo risco. Já o risco de viés moderado compreende que todos os domínios apresentaram risco baixo ou moderado. Se pelo menos um dos domínios apresentou risco severo, e nenhum domínio apresentou risco crítico, então o risco geral é considerado severo também. Por fim, quando pelo menos um domínio apresenta risco crítico de viés, o risco geral é considerado crítico. Quando não há informação suficiente para avaliar o risco de viés, tanto dos domínios quanto do risco geral, diz-se que a avaliaçáo se encontra sem informaçáo. Mais detalhes acerca da definição dos riscos em cada domínio e do processo de avaliação podem ser encontrados na ferramenta ROBINS-I e seu guia de utilização ${ }^{18}$.

A análise estatística dos dados coletados foi realizada com o programa RStudio versão 1.3.959, por meio do método de Mantel-Haenszel. Para cada estudo, a proporção de pacientes que desenvolveram recorrência do CHC com intervalo de confiança de $95 \%$ foi calculada. O risco relativo (RR) foi calculado segundo o modelo de efeitos aleatórios de DerSimonian e Laird. A heterogeneidade entre os estudos foi avaliada pelo índice de inconsistência $\left(\mathrm{I}^{2}\right)$, com valores $>50 \%$ sendo consistentes com a possibilidade de heterogeneidade substancial. P foi considerado significativo quando $<0,05$.

Desse modo, os resultados foram sintetizados e apresentados em escrita narrativa contendo as características dos estudos e a síntese de resultados.

\section{RESULTADOS}

O caminho cursado para a seleção dos estudos iniciou com a pesquisa nas bases de dados com os descritores supracitados, obtendo-se um total de 147 artigos no PubMed, dois artigos no LILACS e 34 artigos na base MEDLINE. Ao excluir os artigos duplicados entre bases $(\mathrm{n}=31)$, restaram 152 artigos, os quais foram analisados de acordo com leitura de títulos e resumo, excluindo-se 138 artigos.

Dos 138 artigos excluídos por intermédio da leitura do título e do resumo, 54 artigos não respondiam à pergunta da pesquisa elaborada e 84 artigos náo obedeciam aos critérios de elegibilidade, sendo 78 por conta do método do estudo (carta ao editor, relato e série de casos, metanálises e estudos de coorte retrospectiva) e seis por conta da ausência de grupo controle.

Quatorze artigos selecionados foram reavaliados integralmente levando em consideração os critérios de elegibilidade e adequaçáo à pergunta da pesquisa e, deste modo, resultaram em sete artigos aplicáveis (Figura 1). 


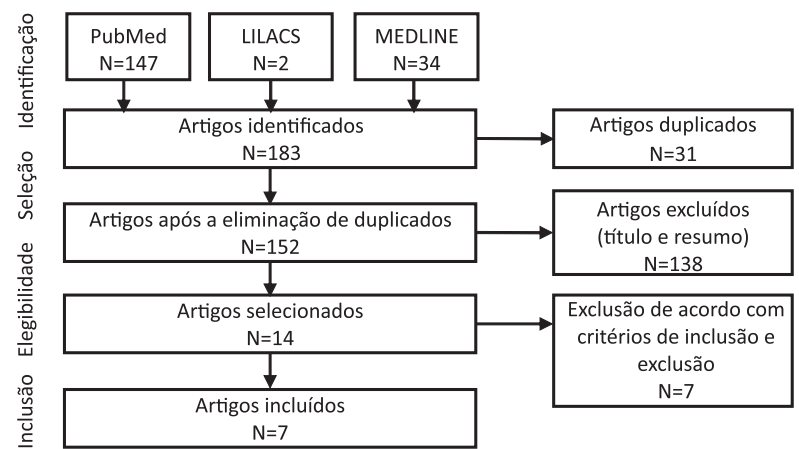

Figura 1. Fluxograma de seleção - PRISMA
Assim, os estudos foram organizados em tabelas e especificados quanto aos autores, ano, título, local, objetivo e metodologia (Quadro 1).

As variáveis descritas em cada estudo bem como os resultados foram detalhados e alocados de maneira comparativa (Tabela 1).

Sendo assim, os sete artigos selecionados compreenderam 847 pacientes. Destes, 452 foram expostos a AAD e 395 adotados como grupo controle. A taxa de recorrência de $\mathrm{CHC}$ em pacientes expostos aos antivirais variou entre $11,1 \%$ a $42,1 \%$, em uma média de

Quadro 1. Características gerais dos estudos incluídos

\begin{tabular}{|c|c|c|c|c|c|}
\hline Autor/ano & Título & Revista & Local & Objetivo & Metodologia \\
\hline Ikeda et al., $2017^{19}$ & $\begin{array}{l}\text { Direct-Acting Antivirals } \\
\text { Decreased Tumor } \\
\text { Recurrence After } \\
\text { Initial Treatment of } \\
\text { Hepatitis C Virus-Related } \\
\text { Hepatocellular Carcinoma }\end{array}$ & $\begin{array}{l}\text { Digestive } \\
\text { Diseases and } \\
\text { Sciences }\end{array}$ & Tóquio, Japão & $\begin{array}{l}\text { Avaliar o impacto da terapia } \\
\text { com AAD sem IFN nas } \\
\text { taxas de recorrência do CHC } \\
\text { relacionado ao } \mathrm{HCV} \text { em } \\
\text { pacientes com histórico de } \\
\text { tratamento curativo do } \mathrm{CHC}\end{array}$ & $\begin{array}{l}\text { Estudo de coorte retrospectivo. } 89 \\
\text { pacientes com histórico de CHC foram } \\
\text { submetidos à terapia com AAD. } 0 \text { tempo } \\
\text { de administração foi de } 12 \text { a } 24 \text { semanas. } \\
\text { Como grupo controle, } 89 \text { pacientes não } \\
\text { receberam nenhuma terapia }\end{array}$ \\
\hline $\begin{array}{l}\text { Warzyszyńska et al., } \\
2017^{20}\end{array}$ & $\begin{array}{l}\text { Accelerated hepatocellular } \\
\text { carcinoma recurrence rate } \\
\text { after postoperative direct- } \\
\text { acting antivirals treatment } \\
\text { - Preliminary report }\end{array}$ & $\begin{array}{l}\text { Clinical and } \\
\text { Experimental } \\
\text { Hepatology }\end{array}$ & $\begin{array}{l}\text { Warsaw, } \\
\text { Polônia }\end{array}$ & $\begin{array}{l}\text { Observar a influência da } \\
\text { terapia com AAD quanto ao } \\
\text { tempo e na frequência da } \\
\text { recorrência do CHC após a } \\
\text { remissão deste }\end{array}$ & $\begin{array}{l}\text { Estudo do tipo caso-controle. } 51 \\
\text { pacientes infectados por HCV foram } \\
\text { observados. Destes, } 19 \text { pacientes } \\
\text { receberam terapia com AAD e } 32 \text { não } \\
\text { receberam esquema antiviral }\end{array}$ \\
\hline Zanetto et al., $2017^{21}$ & $\begin{array}{l}\text { Dropout rate from the } \\
\text { liver transplant waiting list } \\
\text { because of hepatocellular } \\
\text { carcinoma progression in } \\
\text { hepatitis C virus-infected } \\
\text { patients treated with } \\
\text { direct-acting antivirals }\end{array}$ & $\begin{array}{l}\text { Liver } \\
\text { Transplantation }\end{array}$ & Padua, Itália & $\begin{array}{l}\text { Investigar se os pacientes } \\
\text { admitidos com CHC e tratados } \\
\text { com AAD têm uma taxa } \\
\text { aumentada de progressão } \\
\text { tumoral e consequente } \\
\text { abandono da lista de espera } \\
\text { para transplante no Centro de } \\
\text { Transplante de Pádua }\end{array}$ & $\begin{array}{l}\text { Estudo retrospectivo com caso-controle. } \\
46 \text { pacientes foram selecionados; } \\
\text { destes, } 23 \text { pacientes foram submetidos } \\
\text { ao tratamento antiviral e } 23 \text { não } \\
\text { receberam a terapia. } 0 \text { esquema } \\
\text { antiviral adotado foi de } 12 \text { semanas }\end{array}$ \\
\hline $\begin{array}{l}\text { Adhoute et al., } \\
2018^{22}\end{array}$ & $\begin{array}{l}\text { Hepatocellular carcinoma } \\
\text { recurrence in hepatitis } \\
\text { C virus-related cirrhosis } \\
\text { treated with direct-acting } \\
\text { antivirals: A case-control } \\
\text { study }\end{array}$ & $\begin{array}{l}\text { European } \\
\text { Journal of } \\
\text { Gastroenterology } \\
\text { and Hepatology }\end{array}$ & $\begin{array}{l}\text { Barcelona, } \\
\text { Espanha }\end{array}$ & $\begin{array}{l}\text { Investigar o impacto dos AAD } \\
\text { na recorrência do CHC após } \\
\text { terapia com intenção curativa } \\
\text { para CHC }\end{array}$ & $\begin{array}{l}\text { Estudo retrospectivo com caso-controle. } \\
\text { Foram selecionados } 71 \text { pacientes; dos } \\
\text { quais, } 22 \text { receberam a terapia antiviral } \\
\text { e } 49 \text { não a utilizaram. } 0 \text { esquema } \\
\text { utilizado de AAD teve duração de } 12 \text { a } \\
24 \text { semanas }\end{array}$ \\
\hline $\begin{array}{l}\text { Cabibbo et al., } \\
2019^{23}\end{array}$ & $\begin{array}{l}\text { Direct-acting antivirals } \\
\text { after successful treatment } \\
\text { of early hepatocellular } \\
\text { carcinoma improve } \\
\text { survival in HCV--cirrhotic } \\
\text { patients }\end{array}$ & $\begin{array}{l}\text { Journal of } \\
\text { Hepatology }\end{array}$ & $\begin{array}{l}\text { Palermo, } \\
\text { Itália }\end{array}$ & $\begin{array}{l}\text { Estimar se os AAD prolongam } \\
\text { a sobrevida global em } \\
\text { pacientes com cirrose } \\
\text { compensada relacionada ao } \\
\text { HCV e CHC anteriormente } \\
\text { tratado }\end{array}$ & $\begin{array}{l}\text { Estudo retrospectivo com caso-controle. } \\
\text { Foram analisados } 204 \text { pacientes, } 102 \\
\text { receberam terapia antiviral e } 102 \text { não } \\
\text { a receberam. } 0 \text { tempo de terapia foi de } \\
\text { no mínimo } 12 \text { semanas }\end{array}$ \\
\hline Jain et al., $2019^{24}$ & $\begin{array}{l}\text { Is there increased risk of } \\
\text { hepatocellular carcinoma } \\
\text { recurrence in liver } \\
\text { transplant patients with } \\
\text { direct-acting antiviral } \\
\text { therapy? }\end{array}$ & $\begin{array}{l}\text { Hepatology } \\
\text { International }\end{array}$ & Hershey, EUA & $\begin{array}{l}\text { Comparar a taxa de } \\
\text { recorrência de CHC em } \\
\text { receptores transplante de } \\
\text { fígado que receberam terapia } \\
\text { com AAD para aqueles que } \\
\text { não receberam terapia com } \\
\text { AAD e examinar fatores que } \\
\text { podem ter tido impacto na } \\
\text { recorrência do CHC }\end{array}$ & $\begin{array}{l}\text { Estudo retrospectivo com caso-controle. } \\
\text { Foram selecionados } 47 \text { pacientes } \mathrm{HCV} \\
\text { positivos e subdivididos em dois grupos. } \\
0 \text { grupo } \mathrm{A} \text { foi composto por } 27 \text { pessoas } \\
\text { que receberam a terapia antiviral e } \\
\text { o grupo B por } 20 \text { pacientes que não } \\
\text { receberam o tratamento }\end{array}$ \\
\hline Kuo et al., $2020^{25}$ & $\begin{array}{l}\text { The influence of direct- } \\
\text { acting antivirals in } \\
\text { hepatitis C virus related } \\
\text { hepatocellular carcinoma } \\
\text { after curative treatment }\end{array}$ & $\begin{array}{l}\text { Investigational } \\
\text { New Drugs }\end{array}$ & $\begin{array}{l}\text { Taoyuan City, } \\
\text { Taiwan }\end{array}$ & $\begin{array}{l}\text { Elucidar a influência de } \\
\text { agentes AAD na recorrência } \\
\text { de CHC em pacientes com } \\
\text { CHC relacionado ao HCV após } \\
\text { terapias curativas }\end{array}$ & $\begin{array}{l}\text { Estudo retrospectivo com caso-controle. } \\
\text { Os pacientes foram divididos em } \\
3 \text { grupos: tratados com AAD ( } 82 \\
\text { pacientes), tratados como IFN ( } 80 \\
\text { pacientes) e não tratados (160). A } \\
\text { duração do tratamento foi de no } \\
\text { mínimo } 12 \text { semanas }\end{array}$ \\
\hline
\end{tabular}

Legendas: AAD: antivirais de açâo direta; HCV: vírus da hepatite C; CHC: carcinoma hepatocelular; IFN: interferon. 
Tabela 1. Desfechos clínicos dos estudos incluídos

\begin{tabular}{|c|c|c|c|c|c|c|c|c|}
\hline \multirow{2}{*}{$\begin{array}{c}\text { Estudo } \\
\text { Autor/ano }\end{array}$} & \multicolumn{2}{|c|}{ Pacientes (n) } & \multicolumn{2}{|c|}{ Taxa de recorrência (\%) } & \multicolumn{2}{|c|}{$\begin{array}{c}\text { Tempo até diagnóstico } \\
\text { da recorrência } \\
\text { (média de meses) }\end{array}$} & \multirow{2}{*}{$\begin{array}{l}\text { Tempo de } \\
\text { acompanhamento } \\
\text { dos pacientes } \\
\text { (média de meses) }\end{array}$} & \multirow{2}{*}{$\begin{array}{l}\text { Métodos de avaliação } \\
\text { periódica }\end{array}$} \\
\hline & $\begin{array}{l}\text { Grupo } \\
\text { tratado } \\
\text { com AAD }\end{array}$ & $\begin{array}{l}\text { Grupo } \\
\text { controle }\end{array}$ & $\begin{array}{l}\text { Grupo } \\
\text { tratado } \\
\text { com AAD }\end{array}$ & $\begin{array}{l}\text { Grupo } \\
\text { controle }\end{array}$ & $\begin{array}{l}\text { Grupo } \\
\text { tratado } \\
\text { com AAD }\end{array}$ & $\begin{array}{l}\text { Grupo } \\
\text { controle }\end{array}$ & & \\
\hline Ikeda et al., $20171^{19}$ & 177 & 89 & 25 & 46,5 & SI & SI & 20,7 & $\begin{array}{l}\text { TC e/ou RM de abdome a } \\
\text { cada } 3 \text { a } 4 \text { meses }\end{array}$ \\
\hline $\begin{array}{l}\text { Warzyszyńska et } \\
\text { al., } 2017^{20}\end{array}$ & 19 & 32 & 42,1 & 65,6 & 8,8 & 17,7 & SI & $\begin{array}{l}\text { TC e/ou RM de abdome e } \\
\text { dosagem de nível sérico } \\
\text { de AFP em intervalos } \\
\text { trimestrais a semestrais }\end{array}$ \\
\hline $\begin{array}{l}\text { Zanetto et al., } \\
2017^{21}\end{array}$ & 23 & 23 & 12,5 & 8,3 & 7 & 12 & 10 & $\begin{array}{l}\text { TC e/ou RM de abdome } \\
\text { trimestrais no } 1^{\circ} \text { ano } \\
\text { e semestrais após esse } \\
\text { período, dosagem de níveis } \\
\text { séricos de AFP }\end{array}$ \\
\hline $\begin{array}{l}\text { Adhoute et al., } \\
2018^{22}\end{array}$ & 22 & 49 & 41 & 35 & 12 & 14 & 50 & $\begin{array}{l}\text { TC e RM de abdome } \\
\text { trimestralmente durante } \\
\text { os primeiros } 2 \text { anos de } \\
\text { follow-up e semestralmente } \\
\text { após esse período, dosagem } \\
\text { de nível sérico de AFP } \\
\text { trimestral nos primeiros } \\
2 \text { anos de follow-up e } \\
\text { semestralmente após esse } \\
\text { período }\end{array}$ \\
\hline $\begin{array}{l}\text { Cabibbo et al., } \\
2019^{23}\end{array}$ & 102 & 102 & 27,5 & 37,3 & SI & SI & 19,47 & $\begin{array}{l}\text { Exame físico, exames } \\
\text { laboratoriais, US } \\
\text { abdominal trimestral, TC ou } \\
\text { RM dinâmica semestral }\end{array}$ \\
\hline Jain et al., $2019^{24}$ & 27 & 20 & 11,1 & 5 & 9,6 & 10 & 60,85 & $\begin{array}{l}\text { TC de tórax, abdome e } \\
\text { pelve com contraste ou RM } \\
\text { trimestrais }\end{array}$ \\
\hline Kuo et al., $2020^{25}$ & 82 & 160 & 27,5 & 58,8 & SI & SI & SI & $\begin{array}{l}\text { US de abdome e dosagem } \\
\text { de AFP trimestrais }\end{array}$ \\
\hline
\end{tabular}

Legendas: AAD: antivirais de ação direta; SI: sem informação; US: ultrassonografia; TC: tomografia computadorizada; RM: ressonância magnética; AFP: alfafetoproteína.

$26,7 \%$ (desvio-padrão $[\mathrm{DP}] \pm 12,18$ ), contrastando assim com o grupo controle que obteve variação de $5 \%$ a $65,6 \%$, com uma taxa de recorrência média de 36,6\% (DP $\pm 23,2)$.

O tempo até o diagnóstico da recorrência no grupo exposto à terapia foi em média 9,35 meses (mínimo de 7 meses e máximo de 12 meses) com $\mathrm{DP} \pm 1,79)$. Quanto ao grupo controle, a média foi de 13,42 meses (mínimo de dez e máximo de 17,7 meses).

O acompanhamento dos pacientes avaliados em cada estudo sumarizado nesta revisão foi especificado com as seguintes variáveis: tempo de observação, testes clínicos, exames laboratoriais e exames de imagem. O follow-up oscilou entre dez e 60,85 meses, com média de 23,2 meses, aplicando exame físico, provas de imagem (tomografia computadorizada e/ou ultrassonografia abdominal e/ou ressonância magnética) trimestrais, quadrimestrais ou semestrais e dosagem de alfafetoproteína trimestrais ou semestrais.
No Gráfico 1, avaliou-se, por meio de metanálise, o risco de recorrência do $\mathrm{CHC}$ entre os grupos $\mathrm{AAD}$ e controle dos estudos incluídos na revisão. O RR de recorrência do $\mathrm{CHC}$ no grupo de pacientes que recebeu $\mathrm{AAD}$ foi menor do que o risco evidenciado no grupo controle (RR $0,7195 \%$ IC $[0,55 ; 0,93] \mathrm{I}^{2}=38 \%$, $\mathrm{p}=0,14)$.

Por fim, a avaliação do risco de viés foi ponderada de acordo com o protocolo ROBINS- ${ }^{18}$. Tendo sido avaliados todos os domínios, estabeleceu-se um risco de viés geral para cada estudo (Quadro 2).

\section{DISCUSSÃO}

Alguns estudos observacionais sugeriram maior incidência de $\mathrm{CHC}$ e maior risco e agressividade (rápido crescimento e invasão vascular) da recorrência do $\mathrm{CHC}$ após uso da terapia com $\mathrm{AAD}^{9,13}$. A respeito do 


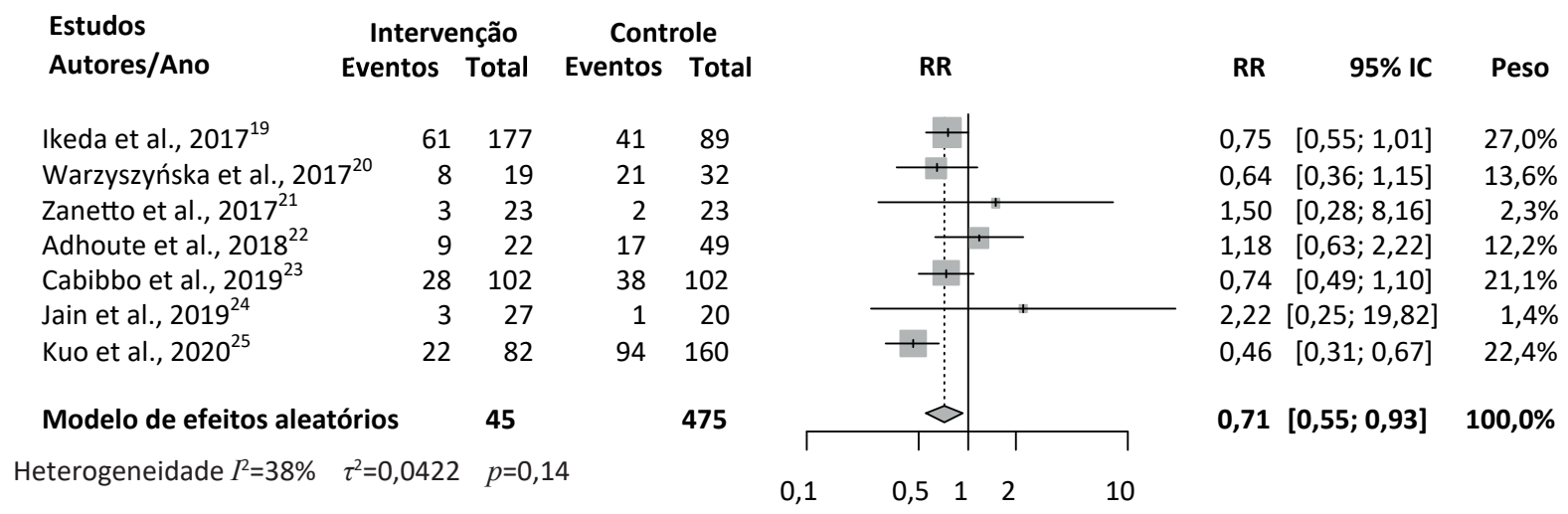

Gráfico 1. Forest plot para avaliar o risco de recorrência do $\mathrm{CHC}$ nos grupos $\mathrm{AAD}$ e controle (pacientes que não receberam nenhuma terapia antiviral)

Legendas: $\mathrm{RR}=$ risco relativo; $\mathrm{IC}=$ intervalo de confiança.

Quadro 2. Avaliação de risco de viés - ROBINS-I

\begin{tabular}{|c|c|c|c|c|c|c|c|c|}
\hline Estudo & \multicolumn{2}{|c|}{ Pré-intervenção } & \multirow{2}{*}{$\begin{array}{c}\text { Intervenção } \\
\text { Classificação de } \\
\text { intervenções }\end{array}$} & \multicolumn{4}{|c|}{ Pós-intervenção } & \multirow[b]{2}{*}{$\begin{array}{l}\text { Risco } \\
\text { de viés } \\
\text { geral }\end{array}$} \\
\hline Autor/Ano & $\begin{array}{l}\text { Domínios de } \\
\text { confusúo }\end{array}$ & Seleção & & $\begin{array}{l}\text { Desvios de } \\
\text { intervenções }\end{array}$ & $\begin{array}{c}\text { Dados } \\
\text { ausentes }\end{array}$ & Desfecho & $\begin{array}{l}\text { Seleção de } \\
\text { resultados } \\
\text { relatados }\end{array}$ & \\
\hline Ikeda et al., $2017^{19}$ & $\odot$ & 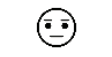 & $\ominus$ & $\odot$ & $\odot$ & 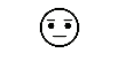 & 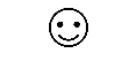 & $\odot$ \\
\hline $\begin{array}{l}\text { Warzyszyńska et al., } \\
2017^{20}\end{array}$ & $\odot$ & $\bigoplus$ & SI & $\odot$ & $\odot$ & $\odot$ & $\odot$ & 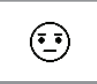 \\
\hline Zanetto et al., 201721 & (2) & $\odot$ & $\odot$ & $\odot$ & $\odot$ & $\odot$ & $\odot$ & $\odot$ \\
\hline $\begin{array}{l}\text { Adhoute et al., } \\
2018^{22}\end{array}$ & 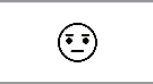 & $\odot$ & $\odot$ & $\odot$ & $\odot$ & $\odot$ & $\odot$ & $\odot$ \\
\hline $\begin{array}{l}\text { Cabibbo et al., } \\
2019^{23}\end{array}$ & $\odot$ & $\odot$ & $\odot$ & $\odot$ & $\odot$ & $\odot$ & $\odot$ & $\odot$ \\
\hline Jain et al., $2019^{24}$ & SI & $\odot$ & SI & $\odot$ & $\odot$ & $\odot$ & $\odot$ & () \\
\hline Kuo et al., $2020^{25}$ & $\odot$ & 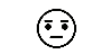 & 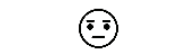 & $\odot$ & $\odot$ & $\odot$ & $\odot$ & $\odot$ \\
\hline
\end{tabular}

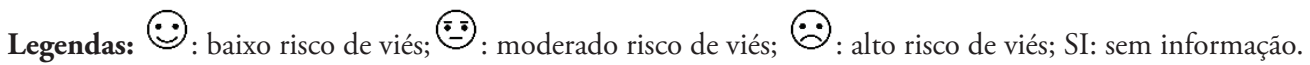

mecanismo fisiopatológico da recorrência inesperada do CHC após o uso de AAD, Villani et al. ${ }^{26}$ descreveram possíveis caminhos. Entre eles, destacam-se disfunção imune, alteraçóes em rede de citocinas e ativação de neoangiogênese causados por mecanismos de açáo dos $\mathrm{AAD}^{26}$.

Com o objetivo de elucidar qual a influência dos AAD na recorrência do $\mathrm{CHC}$, as variáveis de taxa e tempo de recorrência e follow-up presentes nos estudos incluídos nesta revisão foram comparadas com os dados resultantes de outras revisōes sistemática e metanálises, conforme a seguir:

O presente estudo encontrou uma taxa média de recorrência do $\mathrm{CHC}$ de $26,7 \%$ no grupo de pacientes tratados com AAD, em contraste com a taxa média de $36,6 \%$ no grupo controle. O RR de recorrência do $\mathrm{CHC}$ no grupo de pacientes que recebeu $\mathrm{AAD}$ foi menor do que o risco evidenciado no grupo controle (RR 0,71 95\% IC $\left.[0,55 ; 0,93] \mathrm{I}^{2}=38 \%, \mathrm{p}=0,14\right)$. Apesar disso, pontua-se que não houve significância estatística.

Saraiya et al. ${ }^{9}$, em revisão sistemática com metanálise que envolveu 24 estudos publicados, entre janeiro de 2015 e dezembro de 2017 ( $n=1.820$ pacientes), encontraram uma taxa de recorrência média de $24,4 \%$ no grupo de pacientes que recebeu terapia com $\mathrm{AAD}^{9}$. Guarino et al. ${ }^{13}$ também encontraram uma proporção variável semelhante de pacientes tratados com $\mathrm{AAD}$ que desenvolveram uma recorrência do $\mathrm{CHC}$ - de $0 \%$ a $47,9 \%{ }^{13}$.

Reforçando os achados do presente estudo, a metarregressão de Waziry et al. ${ }^{27}$, após ajuste dos estudos incluídos para idade e follow-up, evidenciou que a terapia com $\mathrm{AAD}$ não foi associada à maior recorrência do $\mathrm{CHC}$ 
(RR 0,62, 95\% CI 0,11, 3,45, p=0,56) ${ }^{27}$. Cabibbo et al. ${ }^{23}$ também evidenciaram que a taxa de recorrência do $\mathrm{CHC}$ no grupo de pacientes que recebeu AAD foi inferior quando comparado ao grupo controle, apesar da falta de significância estatística (HR 0,70, 95\% CI $0,44-1,13 ; \mathrm{p}=0,15)^{23}$. Já o estudo de Ikeda et al. ${ }^{19}$ trouxe significância estatística para o mesmo dado, mediante análise multivariada, com hazard ratio de 0,353 (95\% CI $0,191-0,651)$ e $\mathrm{p}=0,001^{19}$. Além disso, a cura da infecção por $\mathrm{HCV}$ com o uso de AAD reduz o risco individual de $\mathrm{CHC}$ de forma semelhante à terapia com IFN. Uma metanálise prévia estimou essa redução em $77 \%$; já a análise de subgrupo de Waziry et al. ${ }^{27}$ apresentou uma diminuição de risco estimada em 63\%.

$\mathrm{O}$ maior risco aparente de incidência e recorrência de $\mathrm{CHC}$ associado ao uso dos $\mathrm{AAD}$ pode ser explicado pela comparação de características de base entre os grupos de pacientes que utilizaram IFN e AAD. Neste último, os pacientes possuíam idade e estágios de cirrose mais avançados - fatores que são preditores do desenvolvimento de $\mathrm{CHC}^{27}$. Isso ocorreu em razão do perfil de segurança $\mathrm{AAD}$, que permitiu que esses medicamentos pudessem ser administrados em pacientes que anteriormente não possuíam indicações para o tratamento com IFN, como, por exemplo, pacientes idosos, com doença hepática avançada (Child-Pugh B e C), coinfectados por HIV, com cirrose descompensada, doença crônica renal severa e transplantados hepáticos ${ }^{10,19,22,28}$. Neste grupo de pacientes, a administração de IFN levava a eventos adversos severos e a uma baixa taxa de eliminação viral ${ }^{19}$. Vale registrar que diversos estudos apontam que não há diferença nas taxas de RVS com uso de AAD em pacientes com idade mais avançada, segundo Pariente et al. ${ }^{29}$. Nesse estudo francês, com pacientes portadores de hepatite $\mathrm{C}$ crônica em uso de AAD, alcançou-se uma RVS de $91 \%$ em uma populaçáo com idade média de 56 anos. Na análise de subgrupos, pacientes com 51-56 anos e com idade superior a 64 anos obtiveram as maiores taxas de RVS ${ }^{29}$. Outro estudo, de Elbaz et al..$^{30}$, que incluiu pacientes com idade média de 65,9 anos, demonstrou uma taxa de RVS de 91,9\% com o uso da combinação sofosbuvir + daclatasvir associada ou não à ribavirina ${ }^{30}$. Contudo, vale lembrar que uma das limitaçốes nesse cenário reside no fato de a maior parte dos estudos de fases II e III excluir pacientes com idade superior a 70-75 anos e com comorbidades severas associadas. Outra constatação importante do estudo francês foi de que a idade por si só não deve ser considerada uma contraindicaçáo ao tratamento com $\mathrm{AAD}$, porém a presença de comorbidades associadas deve ser avaliada, em virtude de uma maior chance de interaçóes medicamentosas negativas e maior desenvolvimento de efeitos adversos ${ }^{29,31}$. Além disso, Keast et al..$^{31}$ evidenciaram que pacientes com hepatite $\mathrm{C}$ crônica tiveram maior falha terapêutica quando utilizavam medicaçóes concomitantes aos AAD (HR 3.218, 95\% CI 1.584-6.530, $\mathrm{p}=0,001$ ). As principais interaçôes significativas apontadas foram entre $\mathrm{AAD}$ e inibidores da bomba de prótons, benzodiazepínicos e inibidores de canais de cálcio ${ }^{31}$. Entre as combinaçôes de agentes AAD disponíveis, um estudo espanhol apontou que a combinação de sofosbuvir e velpatasvir apresenta menor número de interaçóes medicamentosas clinicamente significativas $^{32}$. Essas evidências sugerem a necessidade de realizar uma seleção adequada dos pacientes para realização da terapia com AAD.

Os fatores mais associados com a recorrência do $\mathrm{CHC}$, segundo Saraiya et al. ${ }^{9}$, foram uma história prévia de recorrência do $\mathrm{CHC}$, reportada nesta revisão por Cabibbo et al..$^{23}$ e Ikeda et al. ${ }^{19}$, e um intervalo curto entre a resposta terapêutica completa ao $\mathrm{CHC}$ e o início dos $\mathrm{AAD}$. Outros fatores que podem ser associados com maior taxa de recorrência são níveis séricos elevados de $\mathrm{AFP}, \mathrm{CHC}$ multifocal, doença hepática avançada, invasão vascular microscópica, grau histológico, presença de nodulaçôes satélite, pacientes que receberam quimioembolização transarterial (em inglês, TACE - transarterial chemoembolization - tratamento não curativo, que apresenta maior chance de recorrência) ou outras terapias não curativas, e pacientes que necessitaram de mais de uma intervençáo curativa antes da introdução dos $\mathrm{AAD}^{9,13,22,23}$. Cabibbo et al. ${ }^{23}$ também reforçam em seu estudo que a RVS constitui um importante preditor independente do desfecho nos pacientes que recebem $\mathrm{AAD}$, tanto em termos de recorrência do $\mathrm{CHC}$ quanto em relação à descompensação hepática e principalmente à mortalidade, sendo estatisticamente significativo (HR 0,02, 95\% CI $0-0,19 ; \mathrm{p}<0,001)$. Tal achado torna--se benéfico em um contexto de lista de espera para transplante hepático, no qual o uso do $\mathrm{AAD}$ pode prevenir a saída da lista por progressáo tumoral ou morte dos pacientes. $\mathrm{O}$ autor ainda pontua que o grupo de pacientes que recebeu $\mathrm{AAD}$ apresentou sobrevida global superior ao grupo controle que não recebeu $\mathrm{AAD}$ em seu estudo, sendo estatisticamente significante (HR 0,39, $95 \%$ CI $0,17-0,91, \mathrm{p}=0,03)^{23}$.

O tempo médio entre a introdução da terapia $\mathrm{AAD}$ e o diagnóstico da recorrência foi de 9,35 meses $(\mathrm{DP} \pm 1,79)$. Destaca-se que, entre os autores que trouxeram esses dados, houve uma homogeneidade: daqueles que desenvolveram a recorrência, essa foi mais precoce nos grupos expostos aos antivirais do que o tempo do grupo controle, que observou incidência mais tardia. Adhoute et al. ${ }^{22}$ reportaram que a recorrência do $\mathrm{CHC}$ foi significativamente maior nos pacientes tratados com AAD em intervalo menor do que quatro meses do tratamento prévio de $\mathrm{CHC}$ do que naqueles que tiveram os $\mathrm{AAD}$ introduzidos mais de quatro meses após o tratamento ${ }^{22}$. Saraiya et al. ${ }^{9}$ sugerem ser 
prudente aguardar pelo menos seis meses após a resposta completa ao tratamento do $\mathrm{CHC}$ para introdução dos AAD?.

Saraiya et al. ${ }^{9}$, em suma, expóem resultados heterogêneos, variando conforme o tempo de follow-up. Contudo, o tempo médio apresentado foi de 23,2 meses, sendo superior à média do tempo relatado no presente estudo9. Guarino et al. ${ }^{13}$ descrevem dados concordantes em revisão sistemática publicada em 2018. Entre os resultados, verificou-se que o aparecimento da recorrência foi maior entre os primeiros 36 meses, no entanto, não foi fornecida a distribuiçáo da incidência durante o período citado. Por fim, esses mesmos autores destacam que a frequência da recorrência aumenta conforme o tempo percorrido, aumentando anualmente em $20 \%{ }^{13}$.

Kuo et al. ${ }^{25}$ apontam em seu estudo que os pacientes com CHC apresentam risco tanto para recorrências precoces quanto tardias, sendo que as primeiras estáo mais relacionadas a fatores intrínsecos do tumor, enquanto as segundas se associam mais a fatores relacionados à cirrose, como viremia ativa e severidade da deterioração hepática ${ }^{25}$. Adhoute et al..$^{22}$ também apontaram que as recorrências precoces em um contexto de $\mathrm{CHC}$ podem ter ocorrido em razão de tumores não detectáveis em exames de imagem, usualmente relacionados à disseminaçáo celular antes do tratamento, ou a uma nova ocorrência de um CHC no fígado cirrótico ${ }^{22}$.

Outra possibilidade apontada por Reig et al. ${ }^{33}$, relacionada ao curto intervalo de tempo entre o tratamento prévio de $\mathrm{CHC}$ e a introduçáo dos $\mathrm{AAD}$, diz respeito à interrupçáo abrupta da vigilância imunológica, induzida por esses fármacos com a resolução abrupta do status inflamatório crônico, levando a uma progressão tumoral ${ }^{33}$. No estudo de Reig et al. ${ }^{33}, 27,6 \%$ dos pacientes desenvolveram recorrência tumoral radiológica após um follow-up médio de 5,7 meses $^{33}$.

A média de tempo de acompanhamento dos pacientes na presente revisão foi de 23,2 meses. Saraiya et al. ${ }^{9}$ apresentaram observação por no mínimo três meses e máximo de 36 meses ${ }^{9}$. Do mesmo modo, Guarino et al. ${ }^{13}$ descrevem uma variação entre três meses e 21,6 meses. Sendo assim, o tempo de observação dos pacientes nos estudos analisados na presente revisão mantém-se superior ao das revisóes sistemáticas supracitadas ${ }^{13}$. Quanto à forma de acompanhamento, Saraiya et al. ${ }^{9}$ e Guarino et al. ${ }^{13}$ não apresentaram discordâncias quanto a esse tópico do follow-up, também se fazendo valer de exame físico, exames de imagem e laboratoriais ${ }^{9}, 13$.

Os dados dos estudos incluídos nesta revisão são conflitantes e os estudos apresentam metodologias com limitaçôes. A totalidade dos estudos incluídos nesta revisão são retrospectivos, do tipo coorte ou caso-controle, passíveis de viés de seleção dos pacientes incluídos no estudo. Além disso, os grupos avaliados são quase sempre muito heterogêneos e poucos estudos apresentam análises ajustadas dos dados. Ainda, os estudos mostram medições muito variáveis, como, por exemplo, o tempo até recorrência, que, em alguns estudos, é medido desde o último tratamento curativo para $\mathrm{CHC}$ e, em outros estudos, é considerado a partir do início da terapia com AAD. Sendo assim, os dados apresentam qualidade questionável e, por isso, são necessários estudos com maior rigor metodológico, a fim de diminuir o risco de viés, produzindo assim dados de maior qualidade e confiança.

\section{CONCLUSÃO}

A partir do exposto, sugere-se que a terapia com $A A D$ não aumenta o $\mathrm{RR}$ de recorrência do $\mathrm{CHC}$ em pacientes com infecçáo por HCV. Além disso, quando comparado ao regime terapêutico baseado em IFN, os pacientes se beneficiam de maior eficácia (maior taxa de RVS), menor tempo de tratamento, indicação mais ampla e melhor perfil de tolerabilidade.

A respeito do tempo entre a introduçáo do antiviral e o diagnóstico da recorrência, os resultados apresentados determinaram maior incidência no primeiro ano após a intervenção. Contudo, esse dado pode ser prejudicado pelo tempo de acompanhamento de cada estudo clínico. A somar, entre os pacientes que desenvolveram a recidiva, os estudos analisados mostraram homogeneidade: a incidência foi mais precoce nos pacientes expostos à terapia AAD.

Os procedimentos aplicados para monitorizaçáo dos pacientes foram comuns a todos os estudos analisados e estiveram em concordância com a literatura prévia. Porém, o tempo de follow-up desta revisão destacou-se positivamente quando comparado aos dados já descritos.

Por fim, sugere-se maior produçáo científica que avalie a incidência da recidiva do $\mathrm{CHC}$ a longo prazo e analise o tempo da observação do paciente até o diagnóstico da recorrência.

\section{CONTRIBUIÇÕES}

Todos os autores contribuíram na concepção e/ ou no planejamento do estudo; na obtençáo, análise e interpretaçáo dos dados; assim como na redação e revisão crítica; e aprovaram a versão final a ser publicada.

\section{DECLARAÇÃO DE CONFLITO DE INTERESSES}

Nada a declarar. 


\section{FONTES DE FINANCIAMENTO}

Não há.

\section{REFERÊNCIAS}

1. Ministério da Saúde (BR), Secretaria de Vigilância em Saúde, Departamento de Doenças de Condiçôes Crônicas e Infecçōes Sexualmente Transmissíveis. Hepatites virais 2019. Boletim Epidemiológico. 2019;50(17):5-71.

2. Wong RJ, Gish RG. Metabolic manifestations and complications associated with chronic hepatitis $\mathrm{C}$ virus infection. Gastroenterol Hepatol (NY). 2016;12(5):2939.

3. Axley P, Ahmed Z, Ravi S, et al. Hepatitis C virus and hepatocellular carcinoma: a narrative review. J Clin Transl Hepatol. 2018;6(1):79-84. doi: https:/doi. org/10.14218/JCTH.2017.00067

4. Spârchez Z, Mocan T. Hepatocellular carcinoma occurrence and recurrence after antiviral treatment in HCV-related cirrhosis. Are outcomes different after direct antiviral agents? A review. J Gastrointestin Liver Dis. 2017;26(4)403-10. doi: https://doi.org/10.15403/ jgld.2014.1121.264.hcv

5. Ferlay J, Ervik M, Lam F, et al. Global Cancer Observatory: cancer today [Internet]. Lyon, France: International Agency for Research on Cancer; 2020. Estimated number of incident cases Brazil, both sexes, all ages; 2020 [cited: 2021 Apr 4]. Available from: https://gco.iarc.fr/today/online-analysis-multibars? $\mathrm{v}=2020 \&$ mode $=$ cancer $\&$ mode_population $=$ count ries $\&$ population $=900 \&$ populations $=76 \& \mathrm{key}=$ total $\&$ se $\mathrm{x}=0$ \& cancer $=39$ \& type $=0$ \&statistic $=5$ \& prevalence $=0$ \& population_group=0\&ages_group $\% 5 \mathrm{~B} \% 5 \mathrm{D}=0 \&$ ages group $\% 5$ B $\% 5 \mathrm{D}=17 \& \mathrm{nb} \_$items $=10 \&$ group_ cancer $=1$ \&include_nmsc=1\&include_nmsc_ other $=1$ \&type_multiple $=\% 257 \mathrm{~B} \% 2522$ inc $\% 2522 \% 2$ 53Atrue $\% 252 \mathrm{C} \% 2522$ mort $\% 2522 \% 253$ Afalse $\% 252$ C\%2522prev\%2522\%253Afalse\%257D\&orientation =horizontal\&type_sort=0\&type_nb_items $=\% 257 \mathrm{~B} \% 2$ 522top\%2522\%253Atrue\%252C\%2522bottom\%252 2\%253Afalse $\% 257 \mathrm{D}$

6. Instituto Nacional de Câncer José Alencar Gomes da Silva [Internet]. Rio de Janeiro: INCA; [data desconhecida]. Causas e prevenção: estatísticas de câncer; [modificado 2021 mar 4; acesso 2021 abr 4]. Disponível em: https:// www.inca.gov.br/numeros-de-cancer

7. Sociedade Brasileira de Hepatologia. Consenso sobre hepatite C crônica da Sociedade Brasileira de Hepatologia. São Paulo: Sociedade Brasileira de Hepatologia; 2014.

8. Brunton LL, Hilal-Dandan R, Knollmann BC. As bases farmacológicas da terapêutica Goodman \& Gilman. 13. ed. Porto Alegre, RS: AMGH; 2018.
9. Saraiya N, Yopp AC, Rich NE, et al. Systematic review with meta-analysis: recurrence of hepatocellular carcinoma following direct-acting antiviral therapy. Aliment Pharmacol Ther. 2018;48(2):127-137. doi: https://doi.org/10.1111/apt.14823

10. Boyer TD, Manns MP, Sanyal AJ, editors. Zakim and Boyer's hepatology: a textbook of liver disease. 6th ed. Philadelphia, PA: Saunders/Elsevier; 2011.

11. Rehman S. Safety, tolerability, and associated side effects of direct- acting antivirals, hepatitis C. In: Shahid I, editor. Hepatite C: from infection to cure [Internet]. London: IntechOpen Limited; c2018. doi: https://doi. org/10.5772/intechopen.76225

12. Kwon JH, Yoo SH, Nam SW, et al. Clinical outcomes after the introduction of direct antiviral agents for patients infected with genotype $1 \mathrm{~b}$ hepatitis $\mathrm{C}$ virus depending on the regimens: a multicenter study in Korea. J Med Virol. 2019;91(6):1104-11. doi: https:// doi.org/10.1002/jmv.25412

13. Guarino M, Viganò L, Ponziani FR, et al. Recurrence of hepatocellular carcinoma after direct acting antiviral treatment for hepatitis $\mathrm{C}$ virus infection: literature review and risk analysis. Dig Liver Dis. 2018;50(11):1105-14. doi: https://doi.org/10.1016/j.dld.2018.08.001

14. McGlynn EA, Adams JL, Kramer J, et al. Assessing the safety of direct-acting antiviral agents for hepatitis C. JAMA Netw Open. 2019;2(6):e194765. doi: https:// doi.org/10.1001/jamanetworkopen.2019.4765

15. Yang Y, Wu FP, Wang WJ, et al. Real life efficacy and safety of direct-acting antiviral therapy for treatment of patients infected with hepatitis $C$ virus genotypes 1,2 and 3 in northwest China. World J Gastroenterol. 2019;25(44):6551-60. doi: https://doi.org/10.3748/ wjg.v25.i44.6551

16. Sandmann L, Schulte B, Manns MP, et al. Treatment of Chronic Hepatitis C: efficacy, side effects and complications. Visc Med. 2019;35(3):161-70. doi: https://doi.org/10.1159/000500963

17. Moher D, Shamseer L, Clarke M, et al. Preferred reporting items for systematic review and meta-analysis protocols (PRISMA-P) 2015 statement. Syst Rev. 2015;4(1):1. doi: https://doi.org/10.1186/2046-4053-4-1

18. Sterne JAC, Hernán MA, Reeves BC, et al. ROBINS-I: a tool for assessing risk of bias in non-randomised studies of interventions. BMJ. 2016;355:i4919. doi: https://doi. org/10.1136/bmj.i4919

19. Ikeda K, Kawamura Y, Kobayashi M, et al. Directacting antivirals decreased tumor recurrence after initial treatment of hepatitis c virus-related hepatocellular carcinoma. Dig Dis Sci. 2017;62(10):2932-42. doi: https://doi.org/10.1007/s10620-017-4739-z

20. Warzyszyńska K, Jonas M, Wasiak D, et al. Accelerated hepatocellular carcinoma recurrence rate after postoperative direct-acting antivirals treatment - 
Preliminary report. Clin Exp Hepatol. 2017;3(4):194-7. doi: https://doi.org/10.5114/ceh.2017.71483

21. Zanetto A, Shalaby S, Vitale A, et al. Dropout rate from the liver transplant waiting list because of hepatocellular carcinoma progression in hepatitis $\mathrm{C}$ virus-infected patients treated with direct-acting antivirals. Liver Transpl. 2017;23(9):1103-12. doi: https://doi.org/10.1002/lt.24790

22. Adhoute X, Penaranda G, Raoul JL, et al. Hepatocellular carcinoma recurrence in hepatitis $\mathrm{C}$ virus-related cirrhosis treated with direct-acting antivirals: a case-control study. Eur J Gastroenterol Hepatol. 2018;30(4):368-75. doi: https://doi.org/10.1097/MEG.0000000000001082

23. Cabibbo G, Celsa C, Calvaruso V, et al. Direct-acting antivirals after successful treatment of early hepatocellular carcinoma improve survival in HCV-cirrhotic patients. J Hepatol. 2019;71(2):265-73. doi: https://doi. org/10.1016/j.jhep.2019.03.027

24. Jain A, Miller D, Schreibman I, et al. Is there increased risk of hepatocellular carcinoma recurrence in liver transplant patients with direct-acting antiviral therapy? Hepatol Int. 2019;13(2):190-8. doi: https://doi. org/10.1007/s12072-019-09930-x

25. Kuo YH, Wang JH, Chang KC, et al. The influence of direct-acting antivirals in hepatitis $\mathrm{C}$ virus related hepatocellular carcinoma after curative treatment. Invest New Drugs. 2020;38(1):202-10. doi: https://doi. org/10.1007/s10637-019-00870-9

26. Villani R, Vendemiale G, Serviddio G. Molecular mechanisms involved in HCC recurrence after directacting antiviral therapy. Int J Mol Sci. 2019;20(1):49. doi: https://doi.org/10.3390/ijms20010049

27. Waziry R, Hajarizadeh B, Grebely J, et al. Hepatocellular carcinoma risk following direct-acting antiviral HCV therapy: a systematic review, meta-analyses, and metaregression. J Hepatol. 2017;67(6):1204-12. doi: https:// doi.org/10.1016/j.jhep.2017.07.025

28. Falade-Nwulia O, Suarez-Cuervo C, Nelson DR, et al. Oral direct-acting agent therapy for hepatitis c virus infection: a systematic review. Ann Intern Med. 2017; 166(9):637-648. doi: https://doi.org/10.7326/M162575

29. Pariente A, Arpurt JP, Remy AJ, et al. Effects of age on treatment of chronic Hhepatitis $\mathrm{C}$ with direct acting antivirals. Ann Hepatol. 2019;18(1):193-202. doi: https://doi.org/10.5604/01.3001.0012.7912

30. Elbaz T, Abdo M, Omar H, et al. Efficacy and safety of sofosbuvir and daclatasvir with or without ribavirin in elderly patients with chronic hepatitis $\mathrm{C}$ virus infection. J Med Virol. 2019;91(2):272-277. doi: https://doi. org/10.1002/jmv.25287

31. Keast SL, Holderread B, Cothran T, et al. Hepatitis C direct-acting antiviral treatment selection, treatment failure, and use of drug-drug interactions in a state
Medicaid Program. J Manag Care Spec Pharm. 2019;25(11):1261-67. doi: https://doi.org/10.18553/ jmcp.2019.25.11.1261

32. Sicras Mainar A, Navarro Artieda R, Hernández I, et al. Prevalencia de las potenciales interacciones medicamentosas entre los antivirales de acción directa pangenotípicos y la medicación concomitante asociada a los pacientes con infección del virus de la hepatitis C crónica en Espańa = Prevalence of the potential drugdrug interactions between pangenotypic direct-acting antivirals and the concomitant medications associated with patients with chronic hepatitis $\mathrm{C}$ virus infection in Spain. Gastroenterol Hepatol. 2019;42(8):465-75. doi: https://doi.org/10.1016/j.gastrohep.2019.03.014 Spanish, English.

33. Reig M, Mariño Z, Perelló C, et al. Unexpected high rate of early tumor recurrence in patients with HCV-related HCC undergoing interferon-free therapy. J Hepatol 2016;65(4):719-26. doi: https://doi.org/10.1016/j. jhep.2016.04.008 